\title{
Aspectos neuropsicológicos da esquizofrenia
}

\author{
M iguel A Adad, Rodrigo de Castro e Paulo M attos
}

Universidade Federal do Rio de Janeiro (IPUB)

\section{Introdução}

Pacientes esquizofrênicos demonstram alterações no desempenho em uma grande variedade de testes neuropsicológicos. Estima-se que déficits cognitivos podem ser identificados em $40 \%$ a $60 \%$ dos indivíduos acometidos por essa condição psiquiátrica. Vários estudos clínicos sugerem que alterações cognitivas podem ser observadas desde o início dos sintomas da esquizofrenia. Essas alterações parecem ser características relativamente estáveis na maioria dos pacientes esquizofrênicos, com pequena progressão ao longo do curso da doença. Ainda assim, alguns autores defendem o argumento de que esses déficits cognitivos podem evoluir para um franco processo demencial, pelo menos em certos subgrupos de indivíduos acometidos por essa condição clínica. ${ }^{1}$

Ainda que haja pouca discordância em relação à existência dessas alterações em pacientes esquizofrênicos, não existe unanimidade em relação aos aspectos qualitativos e quantitativos desses déficits. Uma das principais dificuldades nesse sentido é determinada pela grande variabilidade de abordagens metodológicas e conceituais empregadas na investigação dessas alterações. Diferenças em relação à escolha dos grupos de amostragem, instrumentos de avaliação e interpretação dos resultados conduzem frequientemente a conclusões conflitantes sobre esse assunto.

Alguns autores defendem o argumento de que as alterações do desempenho cognitivo observadas na esquizofrenia têm características compatíveis com um padrão de "déficit generalizado", semelhante àquele observado em processos patológicos difusos do SNC (por exemplo, intoxicação por metais pesados, hipoxia cerebral). Esse tipo de argumentação baseia-se principalmente em determinados aspectos do funcionamento cognitivo de indivíduos afetados por essa condição clínica, como tendência à imprecisão, ineficiência e alentecimento das respostas a uma grande variedade de testes neuropsicológicos. ${ }^{2}$

Entretanto, a maioria dos estudos clínicos e experimentais sugere que pacientes esquizofrênicos apresentam alterações mais pronunciadas em relação a determinados setores do funcionamento cognitivo. Os processos de atenção e memória, assim como as chamadas "funções executivas", parecem ser afetados de forma diferenciada em grande parte dos indivíduos acometidos pela doença. ${ }^{3}$ Além disso, pacientes esquizofrênicos tendem a apresentar resultados mais baixos do que a população em geral em baterias de testes psicométricos voltadas à avaliação do quociente de inteligência. ${ }^{4}$ Também são descritas alterações da coordenação motora simples e complexa, além de déficits de linguagem expressiva e receptiva, em indivíduos esquizofrênicos, ainda que essas alterações sejam freqüentemente consideradas secundárias às alterações de atenção, de memória e de funções executivas. ${ }^{3}$

\section{Quociente de inteligência}

Atualmente, o quociente de inteligência é avaliado pela utilização de extensas baterias de testes psicométricos, como a escala WAIS-R (Wechsler Adult Intelligence Scale - Revised). ${ }^{4}$ Essas escalas de avaliação são constituídas por diferentes subtestes, voltados ao exame de processos cognitivos, tais como nível geral de informação, pensamento lógico e abstrato, habilidades verbais e aritméticas, capacidades visoespaciais e viso-construcionais, atenção, memória e destreza psicomotora. No entanto, os resultados dessas escalas devem ser interpretados com cautela, em função das variáveis envolvidas na realização dos diferentes subtestes. Fatores como nível de instrução formal, faixa etária, presença de doença física ou psiquiátrica e utilização de drogas psicoativas podem interferir no desempenho em vários subtestes cognitivos, alterando de maneira significativa o índice final do quociente de inteligência. ${ }^{3,4}$

Estudos clínicos demonstram que o índice do quociente de inteligência pré-mórbido de pacientes esquizofrênicos tende a situar-se em níveis mais baixos do que o de seus parentes em primeiro grau e indivíduos da mesma idade, nível de instrução formal e classe social. O quociente de inteligência pré-mórbido é avaliado por testes padronizados de leitura, como o Nart (National Adult Reading Test), ainda que uma estimativa desse índice também leve em consideração o grau de funcionamento ocupacional anterior ao aparecimento da doença. ${ }^{4} \mathrm{~A}$ utilização de testes de leitura como instrumentos de avaliação do QI prémórbido baseia-se na premissa de que a maioria dos pacientes esquizofrênicos apresenta capacidades de linguagem relativamente preservadas, em contraste com as habilidades não-verbais, que parecem ser mais vulneráveis aos efeitos da doença. ${ }^{4}$

O quociente de inteligência corrente (atual) também é objeto de investigação. Goldberg et $\mathrm{al}^{5}$ realizaram um estudo de avaliação neuropsicológica em pares de gêmeos idênticos discordantes para a esquizofrenia. Esse estudo demonstrou que indivíduos afetados pela doença tendem a apresentar resultados significativamente mais pobres em testes de inteligência do que seus irmãos não afetados, de $80 \%$ a $95 \%$ dos casos. Uma das possíveis implicações desses resultados é que a esquizofrenia 
seria uma condição clínica que impede que o indivíduo atinja o seu nível pleno de desempenho intelectual.

Pacientes esquizofrênicos também parecem apresentar uma redução significativa do quociente de inteligência após o início das manifestações clínicas da doença. Em um amplo estudo realizado por Dunkley \& Rogers, ${ }^{6}$ foi avaliado o desempenho de pacientes esquizofrênicos crônicos hospitalizados em baterias de testes psicométricos direcionadas à avaliação do quociente de inteligência pré-mórbido e corrente. Os resultados obtidos a partir da avaliação desses pacientes indicam que os índices estimados do quociente de inteligência pré-mórbido estavam dentro do padrão de normalidade esperado para a população em geral, ainda que em níveis correspondentes ao desempenho situado na faixa "médio-inferior". Naqueles indivíduos onde o quociente de inteligência corrente pôde ser avaliado, observou-se uma significativa deterioração desse índice, em relação à estimativa do quociente de inteligência pré-mórbido. O declínio intelectual exibido por esses pacientes não estava relacionado à exposição a drogas antipsicóticas ou tratamento prévio pela eletroconvulsoterapia ou insulinoterapia. ${ }^{6}$

\section{Processos de atenção}

Até o final da década de 70, alguns autores como Frith ${ }^{7}$ defendiam o argumento de que as alterações dos processos de atenção seriam os elementos primários de algumas das manifestações clínicas típicas da esquizofrenia, como alucinações auditivas e vivências de influência externa.

Pacientes esquizofrênicos parecem apresentar alterações em relação ao processamento primário de informações, evidenciadas por testes neuropsicológicos voltados à avaliação do tempo de reação. Nüechterlein et $\mathrm{al}^{8}$ demonstraram que pacientes esquizofrênicos tendem a apresentar respostas significativamente mais lentas a estímulos sensoriais auditivos apresentados de maneira seqüencial, em comparação ao desempenho de indivíduos normais pertencentes a grupos de controle. Esse efeito de alentecimento mostrou ser mais pronunciado quando os estímulos eram apresentados de maneira aleatória ou em intervalos muito curtos de tempo. $\mathrm{O}$ alentecimento do tempo de reação parece ser mais pronunciado em tarefas cognitivas em que estímulos são apresentados em diferentes modalidades sensoriais. Em uma série aleatória de estímulos luminosos e auditivos, o tempo de reação torna-se mais longo se o estímulo alvo for precedido de um estímulo de outra modalidade sensorial. Esse efeito de retardo é mais proeminente entre pacientes esquizofrênicos do que em indivíduos pertencentes a um grupo controle. ${ }^{8}$

Pacientes esquizofrênicos também são avaliados em relação à capacidade de aquisição de informações. Em um desses testes, o "Span of Aprehension" (SOA), uma seqüência de até 10 itens (letras, números ou figuras geométricas) é apresentada em curtos intervalos de tempo. Posteriormente, o indivíduo deve identificar os itens anteriormente apresentados na sequiência inicial, a partir de um conjunto maior de elementos. Aproximadamente $40 \%$ dos pacientes esquizofrênicos têm desempenhos abaixo dos níveis encontrados em indivíduos normais.

Pacientes esquizofrênicos também tendem a apresentar resultados mais baixos em relação ao "Digit Span”, um teste rela- tivamente simples, que consiste na repetição verbal de seqüências cada vez maiores de números, até que se estabeleça a quantidade máxima de dígitos a serem repetidos sem erros (o chamado "span" de atenção). O "Digit Span Forward" (direto) é comumente empregado na avaliação de processos de atenção e memória imediata. Em estudo de Oltmanns \& Neale ${ }^{9}$ foram identificadas diferenças entre pacientes esquizofrênicos e indivíduos pertencentes a um grupo de controle em relação ao DSF. Pacientes esquizofrênicos tendem a apresentar mais erros de repetição e "span" de atenção significativamente mais baixo do que indivíduos pertencentes a um grupo controle. ${ }^{9}$

Também parece haver alterações em relação a testes neuropsicológicos elaborados segundo o paradigma "Visual backward masking" (VBM), no qual estímulos visuais são apresentados em intervalos curtos de tempo, sendo rapidamente encobertos por dispositivos automáticos (máscaras). Pacientes esquizofrênicos necessitam de períodos de tempo comparativamente mais longos do que indivíduos pertencentes a um grupo de controle para identificar os estímulos-alvo rapidamente encobertos. ${ }^{10}$

O teste neuropsicológico mais freqüentemente utilizado no estudo das alterações dos processos de atenção em pacientes esquizofrênicos é o "Continuous performance test" (CPT). Nesse tipo de teste neuropsicológico, o indivíduo é solicitado a identificar rapidamente um estímulo alvo entre vários estímulos distratores ou não relevantes. Ainda que baixos rendimentos no CPT possam ser observados em outras condições psiquiátricas, há indicações de que pacientes esquizofrênicos apresentam um padrão específico de alterações nesse tipo de teste. Pacientes esquizofrênicos apresentam tipicamente um comprometimento nos processos de discriminação sinal/ ruído, ainda que não apresentem erros quanto ao critério de identificação. Ainda que crianças portadoras de transtornos de déficit de atenção também possam apresentar comprometimento na discriminação sinal/ ruído, esses indivíduos tendem a apresentar erros freqüentes em relação ao critério de identificação, consistentes com um estilo impulsivo de resposta ao CPT. Alterações do desempenho no CPT na esquizofrenia podem ser observadas mesmo durante períodos de remissão dos sintomas psicóticos. Wohlberg et $\mathrm{al}^{11}$ puderam identificar alterações no desempenho no CPT em pacientes esquizofrênicos clinicamente estáveis em períodos de até um ano após a última hospitalização.

\section{Processos de memória}

Pacientes esquizofrênicos apresentam alterações no desempenho em uma grande variedade de testes de memória. Ainda que alguns autores defendam o argumento de que as alterações de memória na esquizofrenia sejam desproporcionalmente proeminentes em relação a outros distúrbios cognitivos, não existem informações conclusivas a respeito da exata natureza dessas alterações.

Kolb \& Wishaw, ${ }^{12}$ utilizando a Wechsler memory scale (WMS), uma extensa bateria de testes de memória, observaram que pacientes esquizofrênicos tendem a apresentar resultados significativamente mais baixos do que indivíduos do grupo controle em relação às funções de memória verbal e 
visual. A maioria dos estudos voltados à comparação de processos de memória verbal e visual na esquizofrenia encontrou um grau de comprometimento semelhante entre essas duas modalidades de memória. ${ }^{13}$

Em outro estudo clínico, Goldberg et $\mathrm{al}^{13}$ demonstraram que gêmeos monozigóticos discordantes para a esquizofrenia apresentam diferentes padrões de desempenho em baterias de testes de memória. $\mathrm{O}$ desempenho dos pacientes esquizofrênicos é significativamente mais baixo do que aquele obtido por seus irmãos não afetados pela doença, em relação aos mesmos testes de memória. ${ }^{13}$

Entretanto, a maioria dos autores concorda com o argumento de que pacientes esquizofrênicos apresentam alterações das estratégias de organização dos processos de memória. Speed et $\mathrm{al}^{14}$ relataram que pacientes esquizofrênicos apresentam alterações em relação à utilização de semelhanças fonéticas ou semânticas na facilitação dos processos de evocação e aquisição de informações. Resultados semelhantes foram descritos por Manschreck et al, ${ }^{15}$ que relataram uma deficiência na elaboração de estratégias de organização de processos de memória em pacientes esquizofrênicos.

Outro elemento bastante característico do desempenho cognitivo de pacientes esquizofrênicos é a disparidade entre o reconhecimento e a evocação de material previamente aprendido. Em um estudo realizado por Goldberg \& Weinberger ${ }^{16}$ foram avaliados pacientes esquizofrênicos e indivíduos normais pertencentes a um grupo controle, em relação a um teste de memória declarativa, o "Selective reminding test" (SRT). Os indivíduos pertencentes ao grupo controle tiveram um desempenho significativamente melhor em relação à evocação de informações em comparação aos pacientes esquizofrênicos. Em ambos os grupos houve melhora do desempenho nos testes de memória após repetidas tentativas, indicando características de aprendizado semelhantes. Os indivíduos pertencentes ao grupo controle apresentaram desempenhos melhores, em relação ao reconhecimento de material previamente aprendido, do que os obtidos por pacientes esquizofrênicos. Entretanto, pacientes esquizofrênicos exibiram uma grande disparidade entre os processos de reconhecimento e evocação voluntária em comparação ao desempenho de indivíduos pertencentes ao grupo controle. Esses resultados sugerem que pacientes esquizofrênicos têm dificuldades na aquisição de novas informações, ainda que exista um comprometimento mais significativo dos processos de reacessamento de material previamente aprendido. ${ }^{16}$

\section{Funções executivas}

Entre as dificuldades cognitivas mais significativas observadas em pacientes esquizofrênicos estão aqueles processos relacionados às habilidades de abstração e de flexibilidade conceitual envolvidas na solução de problemas. ${ }^{17}$ Essas anormalidades cognitivas têm sido amplamente investigadas por meio da utilização do "Wisconsin card sorting test" (WCST), um teste neuropsicológico freqüentemente empregado na avaliação de processos relacionados a áreas pré-frontais do lobo frontal. ${ }^{19} \mathrm{Um}$ grande número de estudos clínicos e experimentais tem indicado que pacientes esquizofrênicos tendem a apre- sentar desempenhos mais pobres do que a população em geral no WCST. ${ }^{17}$ Esses resultados indicam que pacientes esquizofrênicos apresentam dificuldades significativas em relação à utilização de conceitos abstratos na resolução de tarefas cognitivas. Essas alterações cognitivas freqüentemente conduzem a desempenhos caracterizados por um número elevado de respostas erradas e uma freqüência aumentada de erros de perseveração. Goldberg et $\mathrm{al}^{17}$ demonstraram de maneira consistente que o desempenho no WCST não apresentava melhoras mesmo após instruções explícitas sobre os elementos do teste. Bellack et al, ${ }^{18}$ ao contrário, verificaram melhoras no desempenho de pacientes esquizofrênicos com o WCST, quando do uso de instruções explícitas, apesar dessas melhoras não serem duradouras.

Stuss et al $^{19}$ realizaram um estudo de avaliação de aspectos das funções executivas em pacientes esquizofrênicos e indivíduos normais pertencentes a um grupo de controle, utilizando o WCST como instrumento de avaliação de flexibilidade cognitiva. Esse estudo pôde identificar resultados mais pobres no WCST em pacientes esquizofrênicos, em comparação aos indivíduos do grupo controle.

Em outro estudo semelhante, Levin et $\mathrm{al}^{20}$ puderam comparar o desempenho de pacientes esquizofrênicos, portadores de transtorno bipolar do humor, e indivíduos de um grupo controle, utilizando o WCST como instrumento de avaliação neuropsicológica. Os resultados indicam que pacientes esquizofrênicos tendem a apresentar mais respostas incorretas do que portadores de transtorno bipolar do humor e do que indivíduos normais. Uma análise qualitativa dos erros produzidos durante a realização desse teste mostrou diferentes estratégias de processamento de informações entre os dois grupos de pacientes psiquiátricos. Pacientes esquizofrênicos tendiam a apresentar mais erros de perseveração, enquanto pacientes bipolares apresentavam uma tendência à distraibilidade, tendendo a perder a seqüência adequada de respostas, sugerindo dificuldades de processos de atenção sustentada. ${ }^{20}$

\section{Conclusões}

Existe uma imensa quantidade de informações relacionadas a alterações neuropsicológicas na esquizofrenia. Entretanto, no atual estágio do conhecimento, essas alterações não parecem ser elementos confiáveis como marcadores biológicos da esquizofrenia, principalmente por que não foram estabelecidos padrões específicos de comprometimento cognitivo associados à doença. Esses instrumentos parecem ser de maior utilidade na avaliação do grau de incapacitação interpessoal e ocupacional associado à esquizofrenia. Em um estudo recente de revisão, Green ${ }^{21}$ pôde observar que índices de incapacitação cognitiva eram mais precisos e confiáveis do que a avaliação de sintomas psicóticos per se, no estabelecimento de um prognóstico do funcionamento global.

Os índices de memória verbal no WMS e da flexibilidade cognitiva avaliada pelo desempenho no WCST parecem ser indicadores úteis na previsão da capacidade de interação social (community outcome) em pacientes esquizofrênicos. ${ }^{22}$ 
Os resultados em testes de atenção como o CPT e os índices de desempenho em testes de memória verbal da bateria WMS parecem ser elementos importantes na avaliação das habilidades de solução de problemas sociais (social problem solving). ${ }^{23}$

Um dos principais elementos determinantes do nível de funcionamento interpesssoal e ocupacional é a capacidade de aquisição de habilidades sociais (social skill acquisition). Também foram iden- tificadas relações consistentes entre os resultados de testes de avaliação da memória verbal e de atenção e a capacidade de aquisição de habilidades sociais. ${ }^{24}$

Em recente comunicação, Wykes et al $^{25}$ aliaram as avaliações neuropsicológicas com terapias de recuperação cognitiva e terapia ocupacional intensiva em um grupo de 33 pacientes esquizofrênicos, e obtiveram redução do déficit cognitivo nesses pacientes e conseqüente melhora na adaptação social.

\section{Referências}

1. Meltzer HY, Thompson PA, Lee MA, Ranjan R. Neuropsychologic deficits in schizophrenia: relation to social function and effect of antipsychotic drug treatment. Neuropsychopharmacol 1996;14:28-33.

2. Goldstein G, Zubin J, Pogue-Geile MF. Hospitalization and the cognitive deficits of schizophrenia: the influences of age and education. J Nerv Ment Dis 1991;179:202-6.

3. Gray J, Feldon J, Rawlins J, Hemsley D, Smith A. The neuropsychology of schizophrenia. Behav Brain Sci 1990;14:1-84.

4. Aylward E, Walker E, Bettes B. Intelligence in schizophrenia: metaanalysis of the research. Schizophr Bull 1984;10:430-59.

5. Goldberg TE, Torrey EF, Gold JM, Bigelowl B, Regland RD, Taylor E, et al. Risk of neuropsychological impairment in monozygotic twins discordant and corcordant for schizophrenia. Schizoprh Res 1995; 17:77-84.

6. Dunkley G, Rogers D. The cognitive impairment of severe psichiatric illness: a clinical study. In: David, Cutting, editors. The neuropsychology of schizophrenia. Easy Sussex; 1994.

7. Frith CD. Consciousness, information processing and schizophrenia. Br J Psychiatry 1979;134:225-35.

8. Nuechterlein KH, Dawson ME. Reaction time and attention in schizophrenia: a critical evaluation of the data and theories. Schizophr Bull 1977,3:373-428.

9. Oltmanns TF, Neale JM. Schizophrenic performance when distractors are present: attentional deficit or differentional task dificulty. J Abnorm Psychol 1975;84:205-9.

10.Sacuzzo DP, Braff DL. Information processing abnormalities. Schizophr Bull 1986;12:447-59.

11.Wohlberg GW, Kornetsky C. Sustained attention in remitted schizophrenics. Arch Gen Psychiatry 1973;28:533-7.

12.Kolb B, Wishaw IQ. Performance of schizophrenic patients on tests sensitive to left or right frontal, temporal or parietal lobe function in neurological patients. J Nerv Ment Diseases 1983;171:435-43.

13.Calev A. Recall and recognition in chronic nondemented schizophrenics :the use of matched tasks. J Abnor Psychol 1984;93:172-7.
14.Speed M, Toner BB, Shugar G, Gasbarro II. Thought disorder and verbal and verbal recall in acute psychotic patients. J Clin Psychol 1991;47:735-44.

15.Manschreck TC, Maher BA, Rosenthal JE, Berner J, Reduced primacy and related features in schizophrenia. Schizophr Res 1991;5:35-41.

16.Goldberg TE, Weinberger DR, Pliskin NH, Berman KF, Podd MH. Recall memory deficit in schizophrenia: a possible manifestation of prefrontal dysfunction. Schizoph Res $1989 ; 2(3): 251-7$.

17.Goldberg T, Weinberger DR, Berman KF, Pliskin NH, Podd MH. Further evidence for dementia of the prefrontal type in schizophrenia? Arch Gen Psychiatry 1987;44:1043-51.

18.Bellack AS. Remediation of cognitive deficits in schizophrenia. Am J Psychiatry 1990;147:1650-5.

19.Stuss DT, Benson DF, Kaplan EF, Weir WS, Naeser MA, Liebernan I, et al. The involvment of orbitofrontal cerebrum in cognitive tasks. Neuropsychologia 1983;21:235-49.

20.Levin S, Yegerlun-Todd D. Contribuitions of clinical neuropsychology to the study of schizophrenia. J Abnorm Psychology 1988;4:341-56.

21.Green MF. What are the functional consequences of neurocognitive deficits in schizophrenia? Am J Psychiatry 1996;153:321-30.

22.Lysaker P, Bell M, Beam-Goullet J. WCST and work performance in schizophrenia. Schizophr Res 1995;56:45-51.

23.Corrigan PW, Toomey R. Interpersonal problem solving and information processing in schizophrenia. Schizophr Bull 1995;21:269-81.

24.Mueser KL, Bellack AS, Douglas MS, Wade JH. Prediction of social skill acquisition in schizophrenic and major affective disorder patients from memory and symptomatology. Psychiatr Res 1991;37:281-96.

25.Wykes T, Reeder C, Corner J, Williams C, Everitt B. The effects of Neurocognitive Remediation on Executive Processing with Schizophrenia. Schizophr Bull 1999;25(2):291-307.

Correspondência: Miguel Abib Adad

Rua Jardim Cristofel, 44/21, Independência - CEP 90510-030 Porto Alegre, RS

Fone/fax: (0xx51) 222-1539-E-mail: cejbf@fffcmpa.tche.br 